\title{
Discovery of Apob Small Inhibitor Molecules by Computer Aided Drug Design to Lower Cholesterol Accumulation Based On Docking Analysis
}

\author{
Manas Ranjan Barik ${ }^{1}$, Pinky Sheetal $\mathrm{V}^{2}$ \\ ${ }^{I}$ DNA Labs India, Hyderabad, Ap, India \\ ${ }^{2}$ Department of Bioinformatics, SRM Univeristy, India
}

\begin{abstract}
Cholesterol accumulation leads to coronary heart disease, artherosclerosis, non-insulin dependent diabetes and various other cardiovascular diseases. Apolipoprotein B-100 (ApoB-100) is an integral part of very-low-density lipoprotein $(V L D L)$, intermediate-density lipoprotein $(I D L)$ and low density lipoprotein $(L D L)$, and it plays a key role as a transporter of cholesterol and triacylglycerols to cells throughout the body. Microsomal trigylceride transfer protein (MTP) is a dependent factor as it transfers triglycerides onto lipoprotein particles. The Lipoprotein N-terminal domain (LPD_N)is found in ApoB-100 and MTP. Our study involves the design of suitable small inhibitor molecules to bind to Lipoprotein $N$-terminal domain and inhibit primarily ApoB-100. A library of designed compounds was virtually screened. 14 compounds have been designed that may potentially paralyze its cholesterol transport function. Molecular docking analysis of the ApoB-100 target and designed small inhibitor molecules provides evidence of effective binding of the compounds and interacting residues have been examined. This study could be promising in the discovery of drugs that reduce cholesterol accumulation and therefore result in potential decrease of cardiovascular diseases.
\end{abstract}

Keywords: Apolipoprotein B, ApoB, Cholesterol, Docking, Drug designing

\section{Introduction}

Cholesterol accumulation is an increasing cause for cardiovascular diseases and is currently the major cause of morbidity and mortality. Due the change in lifestyle leading to cholesterol-related diseases such as, atherosclerosis, non-insulin dependent diabetes, obesity, myocardial infarction and coronary heart disease.

ApoB-100 (Apolipoprotein B-100) is part of very-low-density lipoprotein (VLDL), intermediatedensity lipoprotein (IDL) and LDL, and it functions as a transporter of cholesterol and triacylglycerols to cells throughout the body ${ }^{[8]}$. The assembly and secretion of ApoB-containing lipoproteins within the endoplasmic reticulum is strictly dependent on the microsomal trigylceride transfer protein (MTP) which shuttles triglycerides onto the nascent lipoprotein particle ${ }^{[6]}$. Sequence analysis showed that the amino-terminal 700 amino acids of vitellogenin Vtg and apolipoprotein (apo) B-100 are homologous, although the similarity is limited. Coincidentally, Vtg also binds lipids and transports them into the oocytes. The sequence and functional relationship of these two proteins support the idea that they have a common ancestor ${ }^{[2]}$. Like ApoB- 100, vitellogenin binds hydrophobic molecules such as phospholipids, triacylglycerols and cholesterol and it transports these molecules to a target cell. Also, like ApoB-100, vitellogenin binds to a membrane receptor and enters the cell by endocytosis ${ }^{[12]}$.

Computational (in silico) methods have been developed and widely applied to pharmacology hypothesis development and testing. These in silico methods include databases, quantitative structure-activity relationships, similarity searching, pharmacophores, homology models and other molecular modeling, machine learning, data mining, network analysis tools and data analysis tools that use a computer. Such methods have seen frequent use in the discovery and optimization of novel molecules with affinity to a target, the clarification of absorption, distribution, metabolism, excretion and toxicity properties as well as physicochemical characterization ${ }^{[19]}$.

Our study involves the design of a suitable inhibitor molecule to bind to the vitellogenin domain and inhibit MTP and ApoB thereby resulting in potential decreased risk of cardiovascular diseases. By binding to ApoB may paralyze its cholesterol transport function. Bezafibrate is a drug shown to reduce the plasma cholesterol levels in many studies, similarly Miglustat an imino sugar; its treatment reduces lipid storage. Tea catechins ${ }^{[18,7,11]}$ were identified by studies to be responsible for the cholesterol lowering function of green tea and therefore selected for this study. Canadine and berberine, plant alkaloids were identified as cholesterol lowering compounds ${ }^{[17]}$. Significant phytosterols ${ }^{[15,21,10,4]}$ gugglusterone, stanosterol, stigmastan, campestanol and campesterol have been previously studied and shown to have effects in lowering the level of cholesterol and therefore were selected for the designing of potential inhibitor molecules. Molecular docking studies were 
carried out to determine interactionsin order to provide a new insight to design novel molecules that can inhibit the function of the target. The computational analysis was carried out using Discovery Studio (DS) 2.5 (Accelrys Software Inc., San Diego; http://www.accelrys.com).

\section{Materials And Methods}

Cholesterol-related diseases have caused large number of deaths and therefore, in our study this issue has been addressed by designing potent inhibitors in order to help combat the rising health problems associated with cholesterol accumulation.The target APOB_HUMAN (P04114) was retrieved from UniProt ${ }^{[3]}$ and its domains were analyzed in SMART ${ }^{[8]}$.The Lipoprotein $\mathrm{N}$-terminal domain (LPD_N) was found from position 46-598 with an E-value of 6.97e-140. This represents a conserved region found in several lipid transport proteins, including vitellogenin, microsomal triglyceride transfer protein and Apolipoprotein B-100 ${ }^{[1]}$. The target ApoB is then modeled by homology modeling using CPHmodels-3.0 ${ }^{[14]}$ with a Z-score $=59.41$ and validated in DS 2.5.The template chosen was 1LSH- the refined molecular structure of lipovitellin solved at 1.9 $\AA$ having an e-value of $5 \mathrm{e}-05$ and $99 \%$ query coverage when a similarity search by protein-BLAST ${ }^{[20]}$ against Protein Data Bank (PDB) ${ }^{[16]}$ was conducted.

2.1Receptor - Ligands Preparation:

For the current study, bezafibrate, miglustat, canadine, berberine, catechin gallate, epigallocatechin gallate, guggulsterone, stanosterol, stigmastan, campestanol and campesterol were retrieved from PubChem ${ }^{[5]}$ based on various literatures $[15,18,7,11,21,10,4]$ substantiating their biological functionality. Furthermore, CHEMBL143610, CHEMBL141881, CHEMBL140786 and CHEMBL143821 from ZINC ${ }^{[9]}$ database were retrieved for the corresponding target and were also used as scaffold molecules.The lead molecules were designed using ACD/ChemSketch Freeware. The modifications were not made to their functional group maintaining the molecules basic functionality. The 'General Purposes' protocol was used to calculate the molecular properties of the lead molecules to check if the compounds satisfy Lipinski's 'rule of 5'. The target ApoB-100 modeled structure was obtained and the site sphere $(59.8562,11.7025,64.5437,23)$ was defined for the binding site; typing was carried out by CHARMm forcefield (Momany-Rone parital charges methods) and followed by Conjugate Gradient minimization; until a constant potential energy is obtained. The screened compounds were typed similarly.

\subsection{Virtual Screening:}

The aforementioned library of compounds (approximately 222 compounds) was then subjected to Toxicity Prediction (TOPKAT) in the 'ADMET' protocol. NTP Carcinogenicity Call (Male Mouse) (v3.2), FDA Carcinogenicity Female Mouse Single vs Mult (v3.1), Developmental Toxicity Potential (DTP) (v3.1), Rat Oral LD50 (v3.1), Skin Irritation (v6.1) and Aerobic Biodegradabilty (v6.1), were the six criteria selected for the toxicity prediction. Further analysis by ADMET Descriptors in the 'ADMET' protocol was carried out to study the lead compounds pharmacokinetic properties.

2.3 Molecular Docking:

LibDock, a relatively fast algorithm that conducts 'HotSpots' matching of ligand conformations with rigid binding site's HotSpots map that is well-suited for large sized libraries ${ }^{[13]}$, was used to dock the target and ligands into the binding site. The resulting poses with higher LibDock score were investigated and the interacting residues were examined.

\section{Results And Discussion}

Out of the 222 lead compounds designed, most of them qualified Lipinski's 'rule of 5' (Molecular properties shown in Table 1) and their toxicities were studied. Male and Female Mouse Carcinogenicity and Aerobic Biodegradability models were primarily considered for the evaluation of toxicity level. Probability values from 0.0 to 0.30 are considered low probabilities, and are likely to produce a negative response in an experimentalassay; whereas probability values greater than 0.70 are consideredhigh, and are likely to produce a positive response in an experimental assay, probabilities greater than 0.30 but less than 0.70 are considered indeterminate (shown in Table 2). 14 lead molecules with low and intermediate probability values were further investigated for pharmacokinetic properties. The ADMET profiles of the 14 lead compounds are shown in Table 3. Based on the drug-likeness and pharmacokinetic properties these lead molecules were selected for docking and the interactions were studied by docking simulations. The LibDock scores and the binding energies are shown in Table 4. The lead SSr133-[(2E)-2-(3-hydroxycyclohexylidene)ethyl]nonanoic acidderived from stanosterol docked with the highest LibDock score of 121.359and CG383-(benzyloxy)-3,4-dihydro-2Hchromene-6-thiol derived from catechin gallate, docked with a score of 109.827. SSr13 (shown in Fig 1a) interacts with residues LYS209, PRO123 within the lipid transport domain by Intermolecular Hydrogen bonds (Fig 2a) and Bumps (Fig 2b), respectively. CG38 (shown in Fig 1b) interacts with GLY93 by Intermolecular Hydrogen bonds (Fig 3). These compounds have good absorption and high brain concentration/blood concentration level indicated in Fig 4a and Fig 4b. 
Discovery Of Apob Small Inhibitor Molecules By Computer Aided Drug Design To Lower Cholesterol

\begin{tabular}{|l|l|l|l|l|l|l|l|}
\hline Scaffold & Lead ID & ALogP & Mol. Wt & $\begin{array}{l}\text { H- } \\
\text { Acceptor }\end{array}$ & $\begin{array}{l}\text { H- } \\
\text { Donors }\end{array}$ & $\begin{array}{l}\text { Rotatable } \\
\text { Bonds }\end{array}$ & $\begin{array}{l}\text { Aromatic } \\
\text { Rings }\end{array}$ \\
\hline Bezafibrate & BF3 & 4.482 & 393.884 & 5 & 2 & 7 & 2 \\
\hline Canadine & CD18 & 1.992 & 323.407 & 6 & 4 & 7 & 1 \\
\hline 143610 & $143610-1$ & 2.476 & 336.384 & 3 & 3 & 4 & 2 \\
\hline Canadine & CD25 & 2.476 & 219.28 & 3 & 1 & 5 & 1 \\
\hline Canadine & CD26 & 1.385 & 235.279 & 4 & 2 & 6 & 1 \\
\hline Canadine & CD31 & 2.141 & 309.381 & 6 & 3 & 6 & 1 \\
\hline Stanosterol & SSr1 & 4.034 & 196.329 & 1 & 1 & 4 & 0 \\
\hline Stanosterol & SSr12 & 3.914 & 240.382 & 2 & 2 & 7 & 0 \\
\hline Stanosterol & SSr13 & 4.581 & 282.418 & 3 & 2 & 9 & 0 \\
\hline Stanosterol & SSr2 & 2.352 & 198.302 & 2 & 2 & 4 & 0 \\
\hline Stanosterol & SSr3 & 2.487 & 198.302 & 2 & 2 & 4 & 0 \\
\hline $\begin{array}{l}\text { Catechin } \\
\text { Gallate }\end{array}$ & CG21 & 1.644 & 275.3 & 5 & 2 & 5 & 1 \\
\hline $\begin{array}{l}\text { Catechin } \\
\text { Gallate }\end{array}$ & CG37 & 4.034 & 274.742 & 2 & 0 & 3 & 2 \\
\hline $\begin{array}{l}\text { Catechin } \\
\text { Gallate }\end{array}$ & CG38 & 3.729 & 272.362 & 3 & 1 & 3 & 2 \\
\hline
\end{tabular}

Table 1: Molecular properties of the 14 lead molecules; the molecules satisfy Lipinski's 'rule of 5'

\begin{tabular}{|l|l|l|l|l|l|l|}
\hline Molecule & $\begin{array}{l}\text { NTP } \\
\text { Carcinogenicity } \\
\text { Call (Male } \\
\text { Mouse) }\end{array}$ & $\begin{array}{l}\text { FDA } \\
\text { Carcinogenicity } \\
\text { Female }\end{array}$ & $\begin{array}{l}\text { Developmental } \\
\text { Toxicity } \\
\text { Potential }\end{array}$ & $\begin{array}{l}\text { Computed } \\
\text { Rat Oral } \\
\text { LD50 }\end{array}$ & $\begin{array}{l}\text { Skin } \\
\text { Irritation }\end{array}$ & $\begin{array}{l}\text { Aerobic } \\
\text { Biodegradability }\end{array}$ \\
\hline $143610-1$ & 0.026 & 0.011 & 0 & $4.8 \mathrm{~g} / \mathrm{kg}$ & 0 & 0.001 \\
\hline BF3 & 0 & 0 & 1 & $1.5 \mathrm{~g} / \mathrm{kg}$ & 0 & 0 \\
\hline CD18 & 0.036 & 0 & 0.999 & $10 \mathrm{~g} / \mathrm{kg}$ & 0.122 & 0 \\
\hline CD25 & 0.037 & 0 & 1 & $1.5 \mathrm{~g} / \mathrm{kg}$ & 0 & 0.003 \\
\hline CD26 & 0 & 0 & 1 & $1.9 \mathrm{~g} / \mathrm{kg}$ & 0 & 0.26 \\
\hline CD31 & 0 & 0 & 1 & $10 \mathrm{~g} / \mathrm{kg}$ & 0.31 & 0 \\
\hline SSr1 & 0 & 0 & 0 & $10 \mathrm{~g} / \mathrm{kg}$ & 1 & 0 \\
\hline SSr12 & 0 & 0 & 0 & $353.3 \mathrm{mg} / \mathrm{kg}$ & 1 & 0 \\
\hline SSr13 & 0 & 0 & 0 & $10 \mathrm{~g} / \mathrm{kg}$ & 1 & 0.011 \\
\hline SSr2 & 0 & 0 & 0 & $10 \mathrm{~g} / \mathrm{kg}$ & 1 & 0 \\
\hline SSr3 & 0 & 0 & 0 & $10 \mathrm{~g} / \mathrm{kg}$ & 1 & 0 \\
\hline CG21 & 0.476 & 0 & 1 & $9.1 \mathrm{~g} / \mathrm{kg}$ & 1 & 0.161 \\
\hline CG37 & 0.522 & 0.003 & 0.997 & $2.3 \mathrm{~g} / \mathrm{kg}$ & 0 & 0.015 \\
\hline CG38 & 0.004 & 0.004 & 1 & $2.7 \mathrm{~g} / \mathrm{kg}$ & 0 & 0 \\
\hline
\end{tabular}

Table 2: Probability values of the TOPKAT prediction indicate all molecules are non-carcinogenic and have high level of aerobic biodegradability.

\begin{tabular}{|l|l|l|l|l|l|l|}
\hline Lead ID & $\begin{array}{l}\text { BB } \\
\text { level }\end{array}$ & $\begin{array}{l}\text { Absorption } \\
\text { level }\end{array}$ & $\begin{array}{l}\text { Hepatotoxicity } \\
\text { probability }\end{array}$ & $\begin{array}{l}\text { CYP2D6 } \\
\text { Probability }\end{array}$ & $\begin{array}{l}\text { PPB } \\
\text { Level }\end{array}$ & AlogP98 \\
\hline BF3 & 1 & 0 & 0.569 & 0.356 & 2 & 4.482 \\
\hline CD18 & 3 & 0 & 0.463 & 0.415 & 2 & 1.993 \\
\hline 143610-1 & 3 & 0 & 0.582 & 0.504 & 0 & 2.476 \\
\hline CD25 & 1 & 0 & 0.205 & 0.079 & 2 & 2.476 \\
\hline CD26 & 3 & 0 & 0.562 & 0.118 & 2 & 1.385 \\
\hline CD31 & 2 & 0 & 0.543 & 0.425 & 2 & 2.53 \\
\hline SSr1 & 0 & 0 & 0.39 & 0.188 & 1 & 4.034 \\
\hline SSr12 & 1 & 0 & 0.582 & 0.594 & 0 & 3.914 \\
\hline SSr13 & 1 & 0 & 0.509 & 0.455 & 1 & 4.581 \\
\hline SSr2 & 2 & 0 & 0.456 & 0.049 & 0 & 2.353 \\
\hline
\end{tabular}


Discovery Of Apob Small Inhibitor Molecules By Computer Aided Drug Design To Lower Cholesterol

\begin{tabular}{|l|l|l|l|l|l|l|}
\hline SSr3 & 2 & 0 & 0.543 & 0.059 & 0 & 2.487 \\
\hline CG21 & 3 & 0 & 0.39 & 0.405 & 0 & 1.644 \\
\hline CG37 & 0 & 0 & 0.364 & 0.702 & 2 & 4.034 \\
\hline CG38 & 0 & 0 & 0.337 & 0.702 & 2 & 3.729 \\
\hline
\end{tabular}

Table 3: ADMET Descriptors; indicate that the lead compounds are predicted to be easily absorbed, low probability of causing hepatotoxicity and are non - inhibitors of CYPD26 enzyme

\begin{tabular}{|l|l|l|l|l|}
\hline Molecule & $\begin{array}{l}\text { Absolute } \\
\text { Energy } \\
\text { Kcal/mol }\end{array}$ & $\begin{array}{l}\text { LibDock } \\
\text { Score }\end{array}$ & $\begin{array}{l}\text { Intermolecular } \\
\text { Hbonds }\end{array}$ & Intermolecular Bumps \\
\hline 143610-1 & 90.5718 & 109.343 & arg208 & phe1, pro123 \\
\hline BF3 & 62.8112 & 68.2287 & asn110 & phe265, lys171 \\
\hline CD18 & 80.4664 & 86.3169 & - & tyr138, lys171 \\
\hline CD25 & 59.3112 & 106.294 & - & phe1, ile90 \\
\hline SSr1 & 36.4553 & 84.1861 & - & phe1, gly93,val121 \\
\hline SSr12 & 28.3601 & 108.98 & glu92 & phe1, cys43, phe45, lys209 \\
\hline SSr13 & 23.6667 & 121.359 & pro123 & phe1, lys209, \\
\hline SSr2 & 28.8808 & 81.3555 & leu119 & phe1, ile90, gly93 \\
\hline SSr3 & 26.6484 & 87.8954 & leu119 & phe1, pro123, lys209 \\
\hline CG21 & 29.0228 & 67.9289 & - & ile111, val137 \\
\hline CG37 & 34.4242 & 98.921 & gly93, lys209 & - \\
\hline CG38 & 36.1876 & 112.114 & gly93 & - \\
\hline CD26 & 60.2112 & 109.827 & - & lys209, ile90, pro123 \\
\hline CD31 & 74.864 & 82.9614 & lys171 & lys171 \\
\hline
\end{tabular}

Table 4: LibDock scores and binding energies, indicating the residues participating in the binding between receptor and ligand

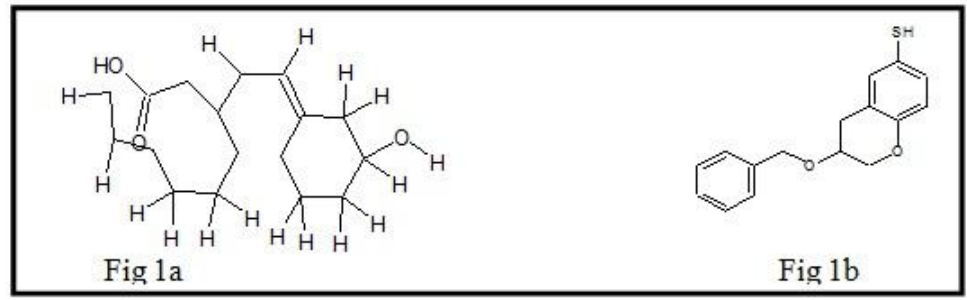

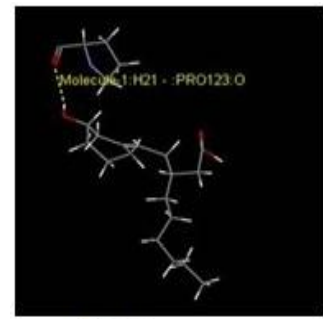

Fig 1a

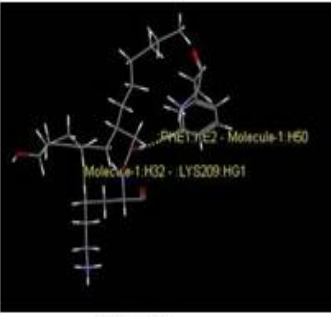

Fig $1 \mathrm{~b}$

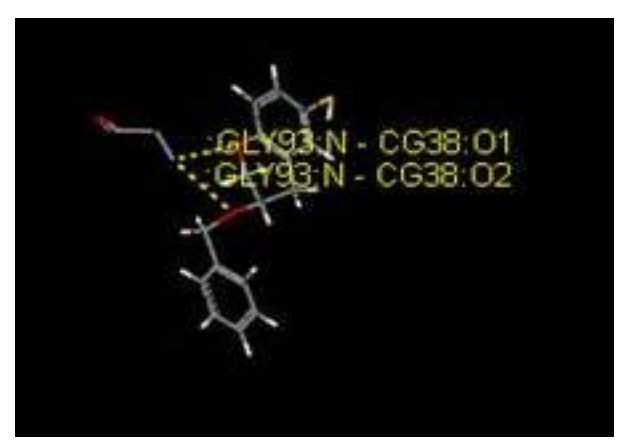

Fig 2a shows Intermolecular Hydrogen bond between SSr13 (H21) and (0-PRO123)target ApoB_LPD_N lipid transport domain. Fig $2 b$ shows Intermolecular Bumps between SSr13 (H32) and (HG1-LYS209) of target ApoB LPD_N lipid transport domain. The interaction with PHE1 is outside the lipid transport domain

ig 3 shows Intermolecular Hydrogen bond between $\mathrm{CG} 38$ (O1 and $\mathrm{O} 2)$ and $(\mathrm{N}-$ GLY93) target ApoB LPD_N lipid transport domain 


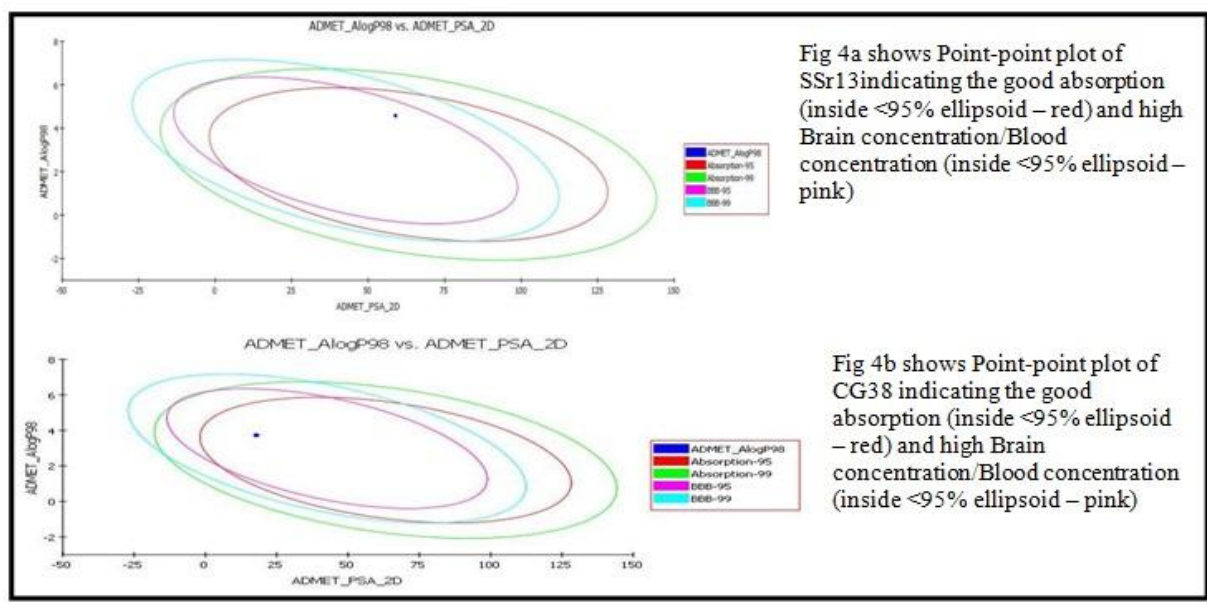

\section{Discussion}

Apolipoprotein B (ApoB) bind to lipid molecules such as cholesterol and triglycerides and transport them and it is primary constituent of chylomicrons and Low Density Lipoproteins that generally contribute to the bad cholesterol in the body leading to various heart diseases. Due this function they play a key role in cholesterol accumulation and have been selected for the current study. The compounds selected as scaffold were phytosterols (guggulsterone, campesterol, campestanol, stanosterol, and stigmastan), tea catechins ( cateching gallate and epigallocatechin gallate), alkaloids ( canadine and berberine) and FDA approved drug (bezafibrate and miglustat) compounds; all proven to reduce the levels of cholesterol.The lead compounds were predicted as non - carcinogenic, non - hepatotoxic, non - inhibitors of the CYPD26 enzyme with good absorption levels and aerobic biodegradability, thus indicating low risk of possible side effects. Molecular docking studies lead to the observation that SSr13 and CG38 bind with highest docking scores thereby indicating potential inhibition of the target ApoB-100 protein. The interacting residues of target, which form hydrogen bonds and bumps with the compounds are ILE90 , GLU92, GLY93, ASN110,ILE111,VAL121,PRO123, VAL137, TYR138,LYS171,ARG208,LYS209, PHE265, lying within the LPD_N lipid transport domain (46 - 598) of the target responsible for the lipid transport.

\section{Conclusion}

Drug discovery process is multi-phased and involves preclinical and clinical trials. Computer aided approach is rapid and significant because it selects thelead molecules with good pharmacological and drug-like properties. ApoB-100 has important role in the utilization of cholesterol, triacylglycerols, and other lipids. The ApoB-containing lipoproteins depend on the microsomal tricylceride transfer protein (MTP) for its secretion. 14 small inhibitor molecules have been designed to bind to the LPD_N lipid transport domain.

Out of the 14 designed inhibitors, 3-[(2E)-2-(3-hydroxycyclohexylidene)ethyl] nonanoic acid (SSr13) designed from the scaffold molecule Stanosterol and 3-(benzyloxy)-3,4-dihydro-2H-chromene-6-thiol (CG38) derived from Catechin gallate was found to be the compounds with best docking scores. Furthermore, the interactions between SSr13 and LPD_Ndomain are more in number suggesting stronger and potentially effective binding. The interacting residues are within the lipid transport domain further substantiating the conclusion that SSr13 is the most potent inhibitor molecule designed to inhibit ApoB-100 and may potentially paralyze its cholesterol transport function.

This study could be a platform for facilitating further development of drugs that moderate cholesterol accumulation and thereby result in likelydecrease of the life threatening cardiacdiseases.

\section{Acknowledgement}

The authors thankfully acknowledge DNA Labs India, Hyderabad for providing all necessary facilities and also grateful to Manas Ranjan Barik for his valuable guidance and assistance in pursuing the research. I would like to thank all the individuals who have contributed for the successful completion of their encouragement and support.

\section{References}

[1] Abdul Hafeez Khan et al., Virtual screening and pharmacophore studies for ftase inhibitors using Indian plant anticancer compounds database, Bioinformation 5(2): 62-66 (2010)

[2] Ankang Li, Murali Sadasivam, and Jeak Ling Ding, Receptor-Ligand Interaction between Vitellogenin Receptor (VtgR) and Vitellogenin (Vtg), Implications on Low Density Lipoprotein Receptor and Apolipoprotein B/E, The Journal of Biological Chemistry,Vol. 278, No. 5, Issue of January 31, pp. 2799-2806, 2003

[3] Cathy H. Wu et al., The Universal Protein Resource (UniProt): an expanding universe of protein information, Nucleic Acids Research, 2006, Vol. 34, Database issue D187-D191 
[4] Christos S. Derdemezis et al., Effects of Plant Sterols and Stanols Beyond Low-Density Lipoprotein Cholesterol Lowering, J CARDIOVASC PHARMACOL THER June 2010 vol. 15 no. 2 120-134

[5] Evan E. Bolton et al., Chapter 12 PubChem: Integrated Platform of Small Molecules and Biological Activities, Annual Reports in Computational Chemistry Volume 4, 2008, Pages 217-241

[6] Greeve J, Inhibition of the synthesis of apolipoprotein B-containing lipoproteins, Handb Exp Pharmacol. 2005;(170):483-517

[7] Ikuo Ikeda et al., Heat-Epimerized Tea Catechins Rich in Gallocatechin Gallate and Catechin Gallate Are More Effective To Inhibit Cholesterol Absorption than Tea Catechins Rich in Epigallocatechin Gallate and Epicatechin Gallate, J. Agric. Food Chem., 2003, $51(25)$, pp 7303-7307

[8] Ivica Letunic et al., SMART 4.0: towards genomic data integration, D142-D144 Nucleic Acids Research, 2004, Vol. 32, Database issue

[9] John J. Irwin and Brian K. Shoichet, ZINC - A Free Database of Commercially Available Compounds for Virtual Screening, J. Chem. Inf. Model., 2005, 45 (1), pp 177-182

[10] Martijn B. Katanet al., Efficacy and Safety of Plant Stanols and Sterols in the Management of Blood Cholesterol Levels, Mayo Clinic Proceedings Volume 78, Issue 8, August 2003, Pages 965-978

[11] Meri P. Nantz et al., Standardized capsule of Camellia sinensis lowers cardiovascular risk factors in a randomized, double-blind, placebo-controlled study, Nutrition Volume 25, Issue 2, February 2009, Pages 147-154

[12] Michael E. BAKER, Is vitellogenin an ancestor of apolipoprotein B-100 of human lowdensity lipoprotein and human lipoprotein lipase?, Biochem. J. (1988) 255, 1057-1060

[13] Mohammad Amine ElGamacy, New inhibitors of VEGFR-2 targeting the extracellular domain dimerization process, Bioinformation 7(1): 52-58 (2011)

[14] Morten Nielsen et al., CPHmodels-3.0 - remote homology modeling using structure-guided sequence profiles, W576-W581 Nucleic Acids Research, 2010, Vol. 38, Web Server issue

[15] Nancy L. Urizar et al., A Natural Product That Lowers Cholesterol As an Antagonist Ligand for FXR , Science 296 (5573): 1703 1706

[16] Nita Deshpande et al., The RCSB Protein Data Bank: a redesigned query system and relational database based on the mmCIF schema, Nucleic Acids Research, 2005, Vol. 33, Database issue D233-D237 